\title{
Isolation of capsulate anaerobic bacteria from orofacial abscesses
}

\section{BROOK}

Armed Forces Radiobiology Research Institute, Bethesda, MD 20814-5145, USA

\begin{abstract}
Summary. The presence of capsulate Bacteroides spp. and anaerobic gram-positive cocci was investigated in pus specimens from 182 children with chronic orofacial infections or abscesses and in pharyngeal swabs from 26 children without inflammation. Of 216 Bacteroides spp. and anaerobic cocci isolated from clinical infections, 170 $(79 \%)$ were capsulate, compared with $34(35 \%)$ of 96 isolates from normal pharyngeal flora $(p<0.001)$. The commonest organisms found to be capsulate more often from infected patients than from controls belonged to the $B$. melaninogenicus group. The possible evolution of encapsulation in these organisms and their importance in mixed orofacial infections are discussed.
\end{abstract}

\section{Introduction}

Aspirates from orofacial infections adjacent to mucous membrane surfaces generally contain a complex bacterial population; the relative importance of the various bacteria isolated is not clear. Several studies have demonstrated the pathogenicity of capsulate anaerobic bacteria, and capsulate strains of Bacteroides spp. and anaerobic grampositive cocci appear to be more virulent than noncapsulated forms of the same organisms (Onderdonk et al., 1977; Brook and Walker, 1983; Brook et al., 1983; Brook et al., 1984; Brook and Walker, $1984 a$ and $b$ ). The pathogenicity of capsulate $B$. melaninogenicus in tonsillitis was also suggested recently (Brook and Gober, 1983).

In the present study the role of capsulate strains of Bacteroides spp. and anaerobic gram-positive cocci in orofacial abscesses and in chronic inflammation of the upper respiratory tract was evaluated by comparing the isolation rate of capsulate organisms for patients with chronic inflammation with the isolation rate of these organisms from the pharynx of normal individuals.

\section{Patients and methods}

\section{Patients}

Specimens were obtained from 182 children with orofacial infections and from 26 children who served as controls. The control group had non-infectious medical conditions, and had not received antimicrobial therapy during the previous month. Both groups were similar in

Received 4 Nov. 1985; accepted 6 Feb. 1986. age, race, and sex. The mean age was 9 years and 2 months (range 3-17 years), and 112 were male.

Forty-eight patients had chronic otitis media, 45 cervical lymphadenitis, 37 chronic sinusitis, 24 chronic mastoiditis, 16 peritonsillar abscesses, and 12 had periapical abscesses. These infectious processes had lasted for at least 10 days before sample collections.

\section{Specimen collection}

Cultures from infected sites were obtained during surgery, which was performed through intact skin (chronic sinusitis and mastoiditis), or by percutaneous aspiration of the abscess cavity after scrubbing the skin or mucous surface with providone-iodine (cervical lymphadenitis, periapical abscess), or by aspiration of an abscess before drainage (peritonsillar abscess), or by aspiration of ear fluid through a perforation in the tympanic membrane (chronic otitis media). Samples were aspirated directly into a syringe that was immediately sealed, or a swab was dipped into the pus and then placed in an anaerobic transport medium (Port-A-Col, BBL Microbiological Systems, Cockeysville, MD). The specimens were transported to the bacteriology laboratory and inoculated on to culture media within 10-30 min after collection.

Cultures from the control group were obtained by swabbing the surface of both tonsils with a sterile cotton swab that was immediately placed in anaerobic transport medium. The specimens were inoculated on to media within 15 min of collection.

\section{Bacteriological procedures}

All cultures were processed for aerobic and anaerobic organisms. Sheep blood (5\%), 'chocolate', and MacConkey Agar plates (BBL, Cockeysville, MD, USA) were inoculated for the isolation of aerobic organisms. The plates were incubated at $37^{\circ} \mathrm{C}$ in an aerobic atmosphere 
(MacConkey) or in air plus $\mathrm{CO}_{2}$ (blood and chocolate), and examined after 24 and $48 \mathrm{~h}$. For anaerobic culture, the specimens were plated on to pre-reduced vitamin $\mathbf{K}_{1}$ enriched Brucella blood agar, a blood-agar plate containing kanamycin $75 \mathrm{mg} / \mathrm{L}$ and vancomycin $7.5 \mathrm{mg} / \mathrm{L}$ and a blood-agar plate containing phenylethyl alcohol, and into enriched thioglycolate broth (Difco Laboratories, Detroit, MI, USA). These media were incubated inside an anaerobic jar (BBL) at $37^{\circ} \mathrm{C}$ and examined after 48 and $96 \mathrm{~h}$. The thioglycolate broth was incubated for 14 days. Aerobic and anaerobic bacteria were identified by conventional methods (Sutter et al., 1980; Lennette et al., 1980). The presence of a capsule in Bacteroides spp. and anaerobic cocci was determined by Hiss's capsule stain (Lennette et al., 1980) and by electronmicroscopy after staining with ruthenium red (Kasper, 1976). Ruthenium red staining demonstrated a homogenous polysaccharide capsule that was external to the cell wall. Statistical analysis was done by $\chi^{2}$ test.

\section{Results}

\section{Abscess contents}

A total of 634 bacterial isolates (426 anaerobes and 208 aerobes) was obtained from the 182 clinical specimens $(2 \cdot 3$ anaerobes and $1 \cdot 1$ aerobes per infected site). The number of isolates from infected sites varied from one to seven. The detailed microbiology of these specimens was similar to previously reported data (Brook, 1980 $a$ and $b, 1981 a, b$ and $c$; Brook et al., 1981) and is therefore not presented here.

\section{Normal pharyngeal flora}

A total of 206 bacterial isolates (112 anaerobes and 94 aerobes) was obtained from the 26 normal pharyngeal specimens ( 4.3 anaerobes and 3.6 aerobes per specimen). B. melaninogenicus and anaerobic gram-positive cocci were isolated from all individuals.

\section{Isolation of capsulate organisms}

Of 216 isolates of Bacteroides spp. and anaerobic gram-positive cocci from clinical infections, 170 $(79 \%)$ were capsulate, compared with $34(35 \%)$ of 96 from normal pharyngeal flora $(\mathrm{p}<0.001)$ (table). The most significant difference was in the rate of encapsulation of the $B$. melaninogenicus group; 67 $(84 \%)$ of the 80 Bacteroides isolates from infected sites were capsulate, compared with $8(23 \%)$ of 35 from controls $(p<0.001)$. Similar but less significant differences were observed with other anaerobic isolates. Analysis of the data according to clinical type of infection also revealed statistically significant differences in the total numbers of capsulate anaerobic isolates from each site of infection (table).

\section{Discussion}

The high isolation rate of capsulate anaerobic bacteria from orofacial abscesses, compared with their prevalence in the normal pharyngeal flora, suggests a pathogenic role for these organisms. Several recent studies have demonstrated the pathogenicity of capsulate anaerobes and their ability to induce abscesses when present alone. Onderdonk et al. (1976 and 1977) correlated the virulence of strains of $B$. fragilis with the presence of a capsule and Simon et al. (1982) described decreased opsonophagocytic killing by neutrophils of capsulate $B$. fragilis. Capsular material from B. melaninogenicus also inhibits phagocytosis by leukocytes and phagocytic killing of other micro-organisms in an in-vitro system (Okuda and Takazoe, 1973). The implication that capsular polysaccharide is associated with virulence is not unique to Bacteroides spp. The capsular material of Streptococcus pneumoniae has been shown to inhibit surface phagocytosis (Wood and Smith, 1949).

In recent studies (Brook et al., 1983 and 1984; Brook and Walker, $1984 a$ and $b$ ) of the relative importance of Bacteroides spp. and anaerobic gram-positive cocci in abscess formation in an animal model, non-capsulate organisms failed to induce abscesses. Many strains with minimal numbers of capsulate organisms $(<1 \%)$, when inoculated with other aerobic and anaerobic bacteria, survived in abscesses and their progeny were capsulate. These capsulate isolates thereafter induced abscesses when injected alone. The selection of capsulate anaerobes occurred in the presence of other capsulate or non-capsulate abscess-forming organisms. This phenomenon may explain how non-capsulate non-pathogenic organisms, which are predominant in the normal flora, can become pathogens. This observation may also explain the importance of anaerobic organisms in chronic infections and in abscess formation, because the process of emergence of capsulate strains took 10 14 days (Brook et al., 1983).

Detection of a capsule in a clinical isolate may indicate that the organism has a pathogenic role in the infection. The demonstration of the importance of capsulate organisms in mixed infections may justify directing therapy in such infections against these potential pathogens. Moreover early and vigorous antimicrobial therapy, directed at the aerobic and anaerobic bacteria present in mixed 


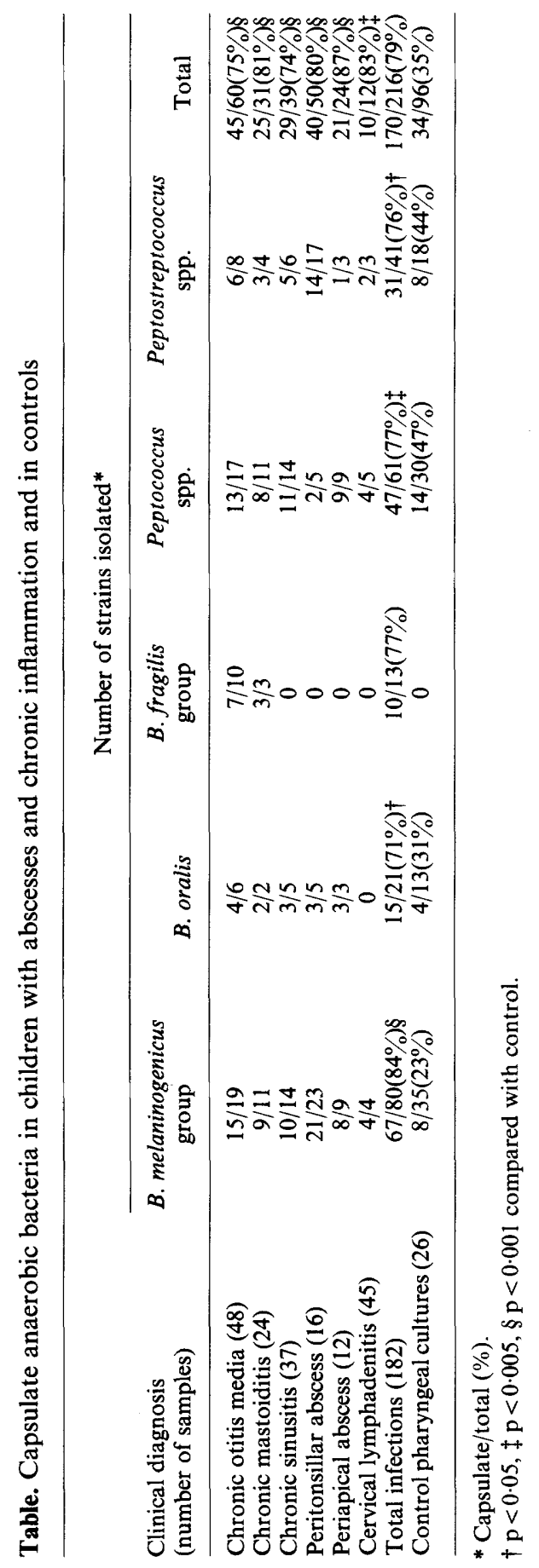


infections, may abort the infection before the capsulate bacteria emerge to contribute to the chronicity of the infection.

The opinions and assertions contained herein are the private ones of the writer and are not to be construed as official or reflecting the views of the Navy Department. the naval services at large, or the Defense Nuclear Agency. The investigations were supported in part by Armed Forces Radiobiology Research Institute. Defense Nuclear Agency, under Research Work Unit MJ 00129.

Research was conducted according to the principles enunciated in the "Guide of the Care and Use of Laboratory Animals" prepared by the Institute of Laboratory Animal Resources, National Research Council. J. E. Perry gave technical assistance. C. H. Dorsey performed the electron-microscopy preparation. and Gloria Contreras gave secretarial assistance.

\section{REFERENCES}

Brook I 1980a Aerobic and anaerobic bacteriology of cervical adenitis in children. Clinical Pediatrics 19:693-696.

Brook $11980 h$ Chronic otitis media in children: microbiological studies. American Journal of Diseases of Children 134:564 566.

Brouk I 198 la Aerobic and anaerobic bacteriology of chronic mastoiditis in children. American Journal of Diseases of Children 135:478 479

Brook I $1981 \mathrm{~h}$ Bacteriologic features of chronic sinusitis in children. Journal of the American Medical Association 246:967 969

Brook I 1981c Aerobic and anaerobic bacteriology of peritonsillar abscess in children. Acta Paediatrica Scandinaria 70:831 835 .

Brook I, Grimm S. Kielich R B 1981 Bacteriology of acute periapical abscess in children. Journal of Endodontics 7:378 380

Brook I 1983 Anaerobic Infections in Childhood. Boston, G.K. Hall.

Brook I. Gillmore J D. Coolbaugh J C. Walker R I 1983 Pathogenicity of encapsulated Bacteroides melaninogenicus group. Bacteroides oralis, and Bacteroides ruminicola subsp. bre'tis in abscesses in mice. Journal of Infection 7:218 226.

Brook I. Walker R I 1983 Infectivity of organisms recovered from polymicrobial abscesses. Infection and Inmunity 42:986 989 .

Brook 1. Gober A E 1983 Bucteroides melaninogenicus: its recovery from tonsils of children with acute tonsillitis. Archites of Otolaryngolog. 109:818-820.

Brook I. Coolbaugh J C. Walker R I 1984 Pathogenicity of piliated and encapsulated Bacteroides fragilis. European Journal of Clinical Microbiolog.' 3:207-209.

Brook I, Walker R I 1984a Pathogenicity of anaerobic grampositive cocci. Infection and Immunity 45:320-324.

Brook I, Walker R I 1984b Significance of encapsulated Bacteroides melaninogenicus and Bacteroides fragilis groups in mixed infections. Infection and Immunity 44:12-15.

Kasper D L 1976 The polysaccharide capsule of Bacteroides fragilis subspecies fragilis: immunochemical and morphologic definition. Journal of Infectious Diseases 133:79-87.

Lennette E H, Balows A, Hausler W J, Truant J P 1980 Manual of clinical microbiology, 3rd edn American Society for Microbiolgy, Washington, D.C.

Okuda K. Takazoe I 1973 Antiphagocytic effects of the capsular structure of a pathogenic strain of Bacteroides melaninogenicus. Bulletin of the Tokyo Dental College 14:99 104.

Onderdonk A B, Bartlett J G, Louie T, Sullivan-Siegler N, Gorbach S L 1976 Microbial synergy in experimental intraabdominal abscess. Infection and Immunity 13:22-26.

Onderdonk A B. Kasper D L, Cisneros D L, Bartlett J G 1977 The capsular polysaccharide of Bacteroides fragilis as a virulence factor: comparison of the pathogenic potential of encapsulated and unencapsulated strains. Journal of Infec'tious Diseases 136:82-89.

Simon G L, Klempner M S, Kasper D L, Gorbach S L 1982 Alterations in opsonophagocytic killing by neutrophils of Bacteroides fragilis associated with animal and laboratory passage: Effect of capsular polysaccharide. Journal of Infectious Diseases 145:72-77.

Sutter V L. Citron D M, Finegold S M 1980 Wadsworth anaerobic bacteriology manual, 3rd edn. C. V. Mosby Co. St Louis.

Wood W B, Smith M R 1949 The inhibition of surface phagocytosis by the capsular 'slime layer' of pneumococcus type III. Journal of Experimental Medicine 90:85-96. 